\title{
Possible usage of cannulated pedicle screws without cement augmentation
}

\author{
Teyfik Demir* \\ Department of Mechanical Engineering, TOBB University of Economics and Technology, Ankara, Turkey
}

\begin{abstract}
.
BACKGROUND: The use of pedicle screws is becoming increasingly popular for spinal surgery practice as the technology advances. Screw pullout due to bone quality and loading conditions is one of the most common problems observed after pedicle screw fixation. Several solutions were studied to prevent screw pullout. These can be investigated under three main categories: screw design, expandable screws and cement augmentation.

OBJECTIVE: This study aimed to investigate the pullout performance of cannulated screws without cement augmentation on synthetic foams.

METHODS: Artificial fusion process for PU is described and validated in our previous studies. For this study six newly designed cannulated pedicle screws were artificially fused to PU foam and pullout test were conducted according to ASTM F543 standard testing protocols.

RESULTS: According to the results of post-fusion pullout tests, worst performed cannulated screw design was $\mathrm{S} 3 \mathrm{H}$ on healthy bone simulating PU foam. However, pullout strength of unilaterally three holes including (S3H) design was purchased with two times higher loads when compared to control group. Solid cored screws were purchased with $671 \mathrm{~N}$ where this value was $1450 \mathrm{~N}$ for $\mathrm{S} 3 \mathrm{H}$ design.

CONCLUSIONS: This study provided that using cannulated pedicle screws without cement augmentation for the cases with healthy bone can be a reliable alternative to classical screws. To the knowledge of the authors this is the first post-fusion study investigating cannulated pedicle screws without cement augmentation.
\end{abstract}

Keywords: Cannulated pedicle screw, pullout, cement augmentation, post-fusion

\section{Introduction}

Pedicle screw insertion is a very common fixation method used for spinal deformities, tumors, vertebral fractures, vertebral infections and degeneration [1]. Bone quality directly effects the bone fractures. Low bone mineral density is more prone to fracture. Even smaller loads can cause bone fractures. Besides, healthy bones (higher bone mineral density) can also be fractured when exposed to higher loads. Both of the broken cases can be fixed and stabilized with pedicle screws.

*Corresponding author: Teyfik Demir, Department of Mechanical Engineering, TOBB University of Economics and Technology, Ankara, Turkey. Tel.: +90 312 2924232; Fax: +90 312 2924091; E-mail: tdemir@etu.edu.tr.
Screw pullout is one of the most common problem following spinal pedicle screw instrumentation. Several solutions were studied to prevent screw pullout. These can be investigated under three main categories: screw design, expandable screws and cement augmentation [2-12]. Design studies on screw core geometry (cylindrical or conical), number of threads, flank overlap area, pitch diameter, dual lead and dual core were investigated by researchers.

Expandable screw seems to be more advantageous than most of the screw types when the comparison carried out on primary biomechanical strength. The contra conical geometry of the screw body after expansion is the most remarkable advantage that offered by expandable screws. However, the most critical challenge on the usage of expandable screw is revision surgeries. Revision became quite impossible due to the new bone 
formation between the expanded screw tip after fusion [8].

Cannulated screws were also studied as alternatives of solid cored or expandable screws. Cement types, position and numbers of radial holes, cement injection technique were also investigated to increase the pullout performance especially for osteoporotic vertebrae. Most of the works focused on the usage of two types of cement. One is calcium based [9, 19-22] and the other is poly-methyl-meta-acrylate (PMMA) [9,13-15]. Researchers have found out that screws with Ca phosphate injection had 3 times higher pullout strength, compared to screws without cement augmentation [16].

Some researchers combined expandable screws and cement augmentation in their studies [6, 15, 17]. Researches indicated that expandable screws with cement augmentation had $43 \%$ improved pullout strength [15] According to Cook et al. [17] expandable screws with cement augmentation had two or three times higher pullout strength than only solid cored screws for osteoporotic cases.

This study investigated the possible usage of cannulated screws without cement augmentation. The main motivation under this phenomenon is the boneingrowths mechanism through radial holes/slots. To understand this effect, previously designed and validated six different types of cannulated screws were used. In addition, a solid cored screw was also tested as a control group. Samples were tested under two main conditions. To determine the primary strength of screw standard solid PU foam blocks were used as testing medium. Secondly, artificial fusion process was applied to all designs and control group to determine the post-fusion pullout strength.

\section{Materials and method}

\subsection{Design parameters}

Six different cannulated screw designs were used in this study. In addition, Solid cored classical pedicle screw with same geometrical features and thread/core type of cannulated screws was also tested as control group. Figure 1 depicts the design parameters for screws and the denotations. All cannula diameters were $2 \mathrm{~mm}$ and radial holes were drilled with a diameter of $1.5 \mathrm{~mm}$. Slots were milled with a $2 \mathrm{~mm}$ diameter end-mill cutter. All holes and slots were drilled/milled unilaterally and bilaterally. Hole and slot types are given in Fig. 2. All designed screws were having $6 \mathrm{~mm}$ outer diameter and $45 \mathrm{~mm}$ length and made of Ti alloy namely, Ti6Al4V [18]. On the designation of designed screws first capital letter represents the unilaterality (S) or bilaterality (D) of the holes and slots. Additionally,

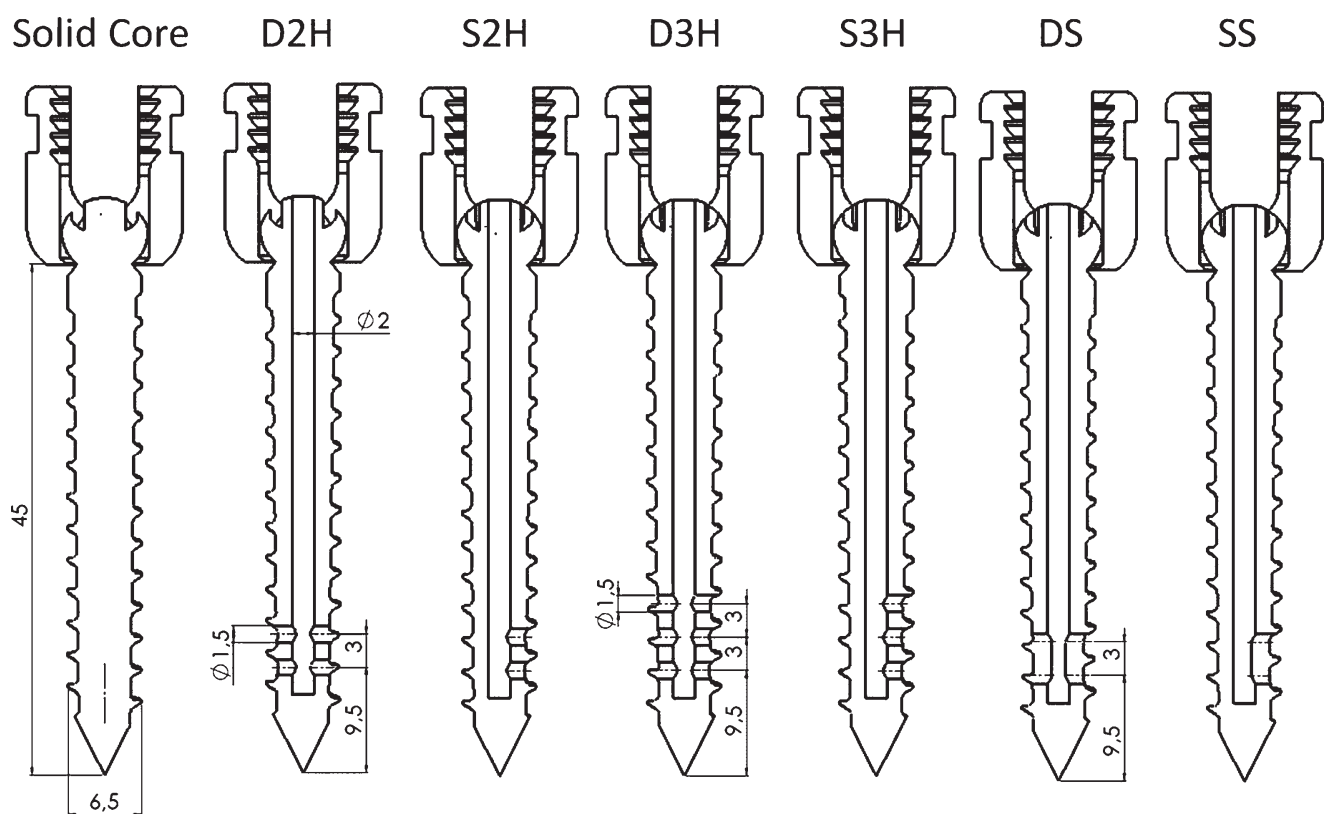

Fig. 1. Design parameters of cannulated screws. 

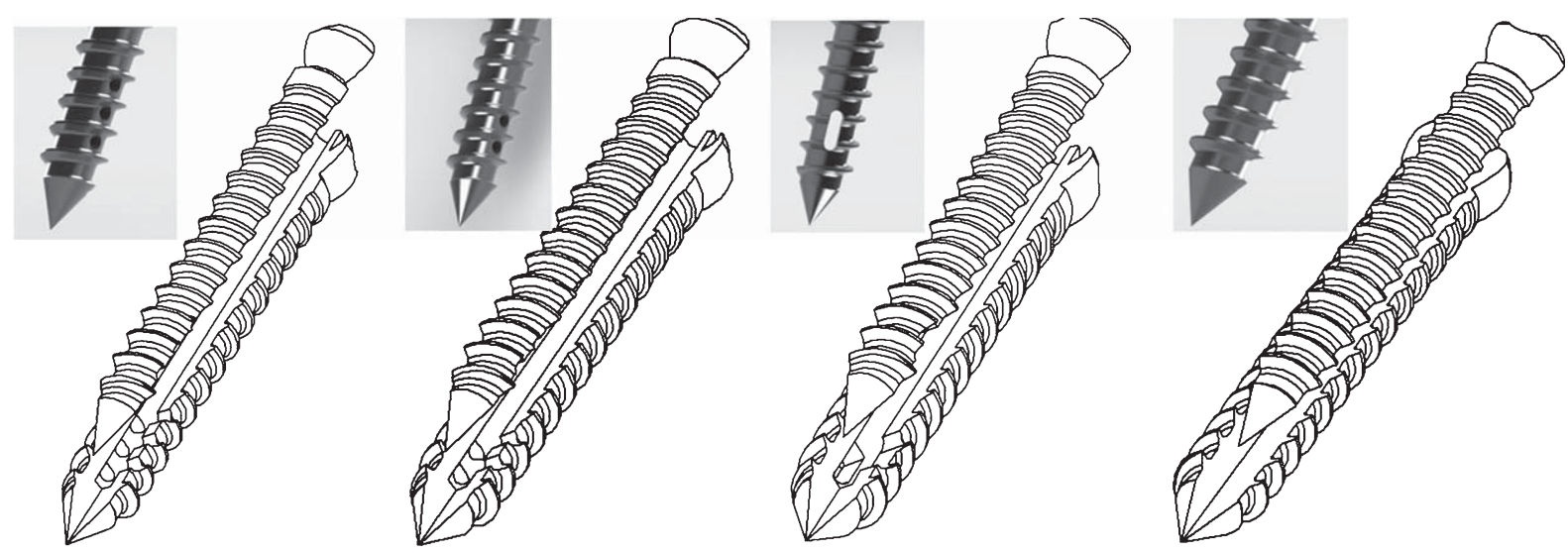

Fig. 2. Cross section and illustration of hole/gap types. Three holes, two holes and slot including designs with solid cored screw design. (From left to right).

$2 \mathrm{H}, 3 \mathrm{H}, \mathrm{S}$ represents the two holes, three holes and slot, respectively.

\subsection{Embedding medium and artificial fusion process}

Embedding medium was synthetic foam. Polyurethane (PU) foam is standard testing material for orthopedic implants. Different grades of PU foam were described in ASTM F1839 [19]. Two different grades of PU foam were used in tests. Grade $40 \mathrm{PU}$ foam was used as healthy bone simulating/mimicking material. Similarly, Grade 10 PU foam was used as osteoporotic bone simulating material. Mechanical properties of the PU foams were briefly described in Table 1. Additionally, PU foam was used in two different forms. First, solid foam blocks were as used as all standard applications. After the insertion of each cannulated screw $2 \mathrm{ml}$ PMMA cement was injected with appropriate hand tool. Second, a new technique was applied namely, artificial fusion process. This process was firstly raised by Arslan et al. [20] to understand the effects of radial holes on pedicle screw pullout strength after the fusion. Same method of artificial foaming process was used in this study.

Table 1

Mechanical properties of polyurethane foam

\begin{tabular}{lcccc}
\hline & $\begin{array}{c}\text { Density } \\
\left(\mathrm{g} / \mathrm{cm}^{3}\right)\end{array}$ & Standard dev. & $\begin{array}{c}\text { Compressive } \\
\text { strength }(\mathrm{MPa})\end{array}$ & Standard dev. \\
\hline Grade 10 & 0.16 & 0.005 & 2.5 & 0.2 \\
Grade 40 & 0.64 & 0.01 & 35 & 0.5 \\
\hline
\end{tabular}

Polyurethane contains two components. One is polyol and the other is polyisocyanate. On the production of polyurethane foam mixing these two components is the main step. When one mixed these two components of polyurethane mix starts to foam with an exothermic reaction. In our study, after mixing these two components with appropriate ratios in a close volume die, screws were inserted to the die just before the foaming process as shown in Fig. 3. This procured the bone in growth simulation through the radial holes/slots of the cannulated screws. Curing time of the mixture was 20 minutes; however tests were performed 24 hours after the curing process [20].

\section{Experimental procedure}

Pullout Tests: Pullout test setup is given in Fig. 4. PU blocks with inserted screws were placed to test setup as described in figure. Pullout tests were carried out to the screws according to the ASTM F543[21] standard testing protocols. Screws were embedded in to the foam $30 \mathrm{~mm}$ from the distal end (tip). Tests were performed with Instron 5569 testing frame (UK). Pullout rate (cross head speed) was $2 \mathrm{~mm} / \mathrm{min}$. Load versus displacement data was recorded during the tests. The failure criterion was the threshold of the pullout force over than $80 \%$ (pullout occurrence).

After completing the tests statistical analysis were performed on test results. Student- $t$ test was applied to understand whether the differences between two groups are significant or not. Statistical comparison is given in Table 2. 

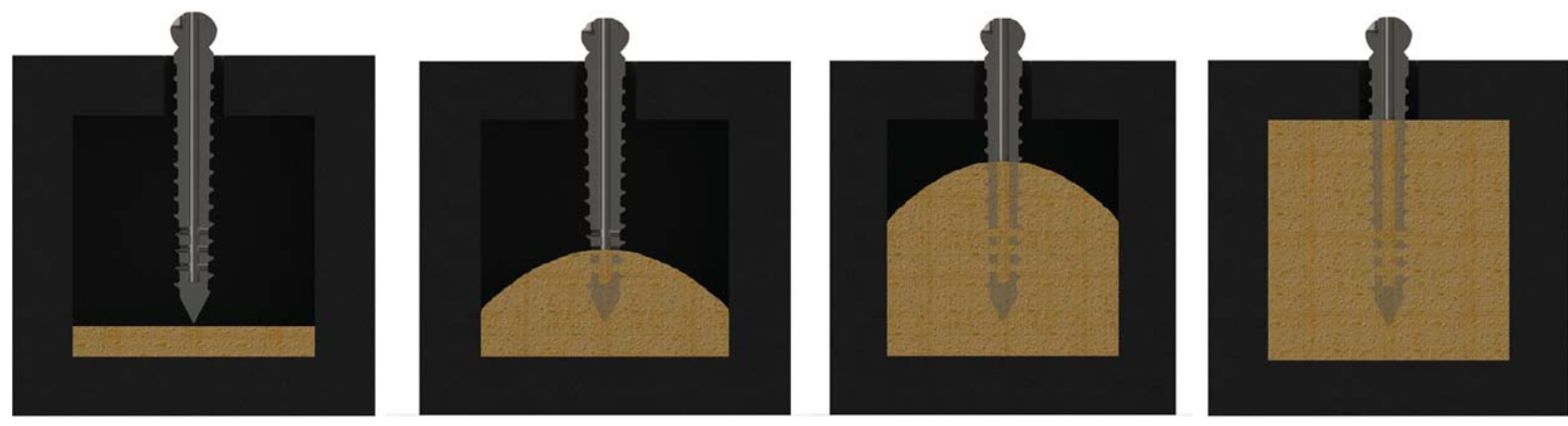

Foaming Process

Fig. 3. Foaming Process: Simulation of bone fusion.

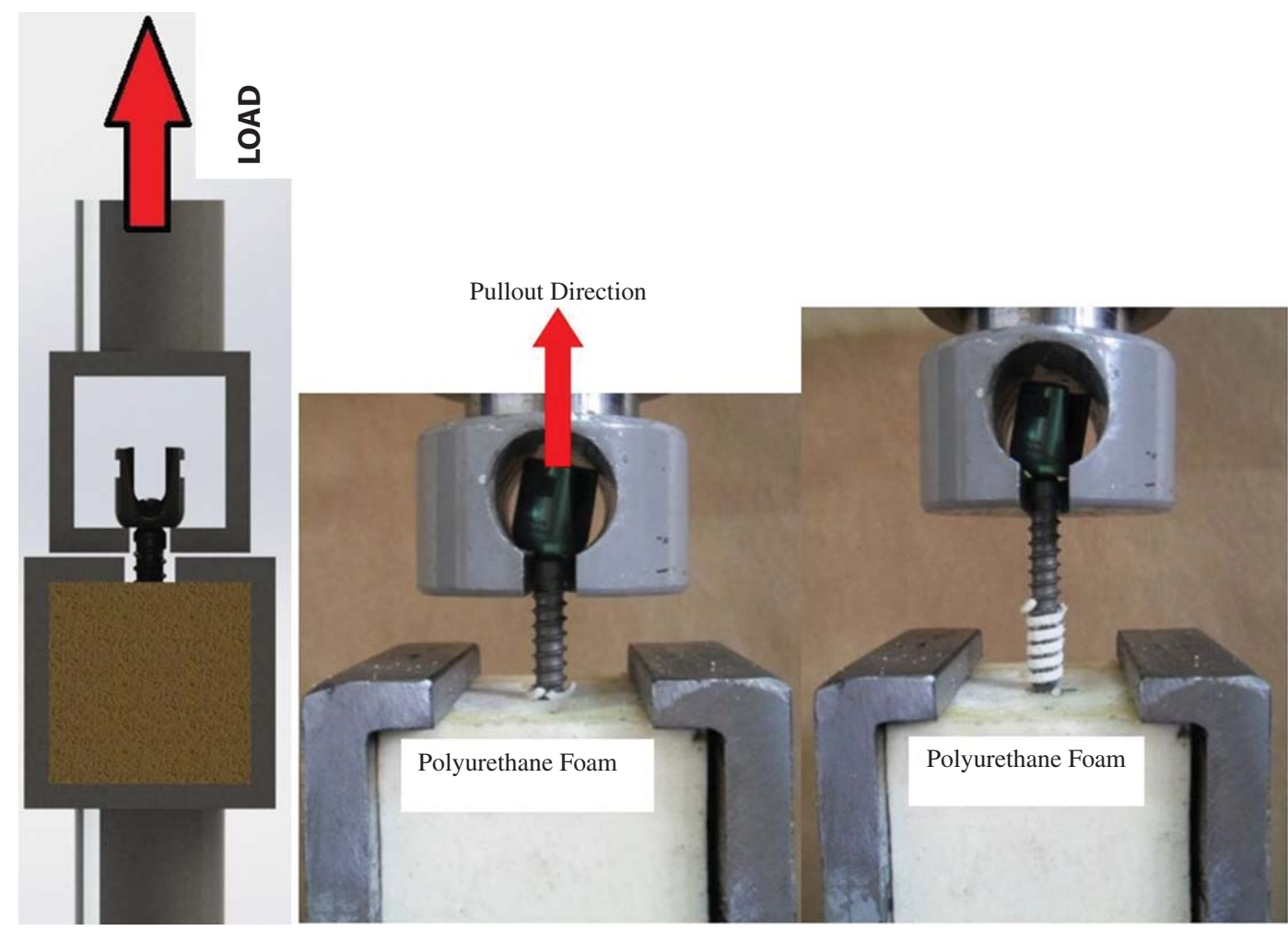

Fig. 4. Pullout test setup. From left to right; Schematic view, before pullout and after pullout, respectively.

\section{Results}

Primary fixation (without fusion occurrence) and post fusion pullout strength comparison is given in Table 3. According to the test results, bilateral hole/slot including designs were exhibited close to two times higher pullout resistance than unilateral hole/slot including designs among all designed screws when the comparison carried out on Grade 40 PU foam. For instance, maximum pullout forces were $1586 \mathrm{~N}$ and $2784 \mathrm{~N}$ for S2H and D2H screws, respectively. Similarly, maximum pullout force difference between D3H and $\mathrm{S} 3 \mathrm{H}$ was $1931 \mathrm{~N}$ while the pullout strength of $\mathrm{S} 3 \mathrm{H}$ was $1450 \mathrm{~N}$. In addition, maximum pullout strengths were $2228 \mathrm{~N}$ and $3031 \mathrm{~N}$ for SS and DS screws, respectively. 
Table 2

Statistical comparison of pullout test results for different designs

\begin{tabular}{|c|c|c|c|}
\hline \multicolumn{2}{|c|}{ Grade 40} & \multicolumn{2}{|c|}{ Grade 10} \\
\hline Comparison & $p$ values & Comparison & $p$ values \\
\hline Solid Core \& S2H & $0.00716^{*}$ & Solid Core \& S2H & $0.00303^{*}$ \\
\hline Solid Core \& D2H & $0.00033^{*}$ & Solid Core \& D2H & $0.00167^{*}$ \\
\hline Solid Core \& S3H & $0.01140^{*}$ & Solid Core \& S3H & $0.00332^{*}$ \\
\hline Solid Core \& D3H & $0.00000^{*}$ & Solid Core \& D3H & $0.00023^{*}$ \\
\hline Solid Core \& SS & $0.00517^{*}$ & Solid Core \& SS & $0.00040^{*}$ \\
\hline Solid Core \& DS & $0.00000^{*}$ & Solid Core \& DS & $0.00089^{*}$ \\
\hline $\mathrm{S} 2 \mathrm{H} \& \mathrm{D} 2 \mathrm{H}$ & $0.00156^{*}$ & S2H \& D2H & 0.20388 \\
\hline $\mathrm{S} 2 \mathrm{H} \& \mathrm{~S} 3 \mathrm{H}$ & 0.59811 & $\mathrm{~S} 2 \mathrm{H} \& \mathrm{~S} 3 \mathrm{H}$ & 0.71678 \\
\hline $\mathrm{S} 2 \mathrm{H} \& \mathrm{D} 3 \mathrm{H}$ & $0.00023^{*}$ & $\mathrm{~S} 2 \mathrm{H} \& \mathrm{D} 3 \mathrm{H}$ & 0.41399 \\
\hline S2H \& SS & 0.09447 & S2H \& SS & 0.86235 \\
\hline S2H \& DS & $0.00042^{*}$ & S2H \& DS & 0.60925 \\
\hline $\mathrm{D} 2 \mathrm{H} \& \mathrm{~S} 3 \mathrm{H}$ & $0.00073^{*}$ & $\mathrm{D} 2 \mathrm{H} \& \mathrm{~S} 3 \mathrm{H}$ & 0.34793 \\
\hline D2H \& D3H & $0.02717^{*}$ & D2H \& D3H & $0.01454^{*}$ \\
\hline D2H \& SS & 0.13961 & D2H \& SS & 0.12560 \\
\hline D2H \& DS & 0.28109 & D2H \& DS & 0.05079 \\
\hline $\mathrm{S} 3 \mathrm{H} \& \mathrm{D} 3 \mathrm{H}$ & $0.00014^{*}$ & S3H \& D3H & 0.20938 \\
\hline S3H \& SS & $0.04995^{*}$ & S3H \& SS & 0.78704 \\
\hline S3H \& DS & $0.00023^{*}$ & S3H \& DS & 0.36084 \\
\hline D3H \& SS & $0.01590^{*}$ & D3H \& SS & 0.17105 \\
\hline D3H \& DS & $0.02357^{*}$ & D3H \& DS & 0.74448 \\
\hline SS \& DS & $0.04291^{*}$ & SS \& DS & 0.39423 \\
\hline
\end{tabular}

* Statistically significant difference $(p<0.05)$.

According to the results of post-fusion pullout tests, worst performed cannulated screw design was $\mathrm{S} 3 \mathrm{H}$ on healthy bone simulating PU foam. However, pullout strength of $\mathrm{S} 3 \mathrm{H}$ design was purchased with two times higher loads when compared to control group $(P<0.05)$. Solid cored screws were purchased with $671 \mathrm{~N}$ where this value was $1450 \mathrm{~N}$ for S3H design.

Contrary to the tests carried out with grade $40 \mathrm{PU}$ foam, cannulated screws without cement augmentation exhibited similar performance between straddle and bilateral hole/slot including designs when embedding medium is osteoporotic (Grade $10 \mathrm{PU}$ foam). S2H and D2H screws were purchased with $978 \mathrm{~N}$ and $787 \mathrm{~N}$ pullout forces $(P=0.20)$, respectively. Similar to this,
S3H, D3H, SS and DS screw designs were also purchased with pullout force of $919 \mathrm{~N}, 1095 \mathrm{~N}, 954 \mathrm{~N}$ and $1057 \mathrm{~N}$, respectively. There were no significant differences between groups (Table 2).

Similar to Grade 40 tests, pullout strength of solid cored screw was three times lower than the worst performed cannulated screw design. The minimum pullout force was $787 \mathrm{~N}$ for D2H screws. This results were obtained from Grade 10 PU foam (osteoporotic case) used tests after artificial fusion process. Solid cored screw exhibited $236 \mathrm{~N}$ pullout strength after fusion in osteoporotic synthetic foam material.

\section{Discussion}

Pullout tests that conducted on solid PU foam blocks were the simulation of early stage pullout phenomenon. On the other hand, artificially fused PU cases were the simulation of post fusion performances of the designed screw. One should be aware that early stage pullout is still very important problem. However, the results of post-fusion tests are the scenario of further stages of fixation. Fusion generally takes 3-5 months for spinal fusion surgery patients. This study's main focus is on the possible usage of cannulated screws without cement augmentation for fusion occurred cases. To state the difference between primary fixation performance and post fusion cases two embedding medium with two different bone qualities were used in tests.

The main advantage of the cannulated screws is the prevention of the pullout for osteoporotic cases. In addition, this study focused on possible usage of cannulated pedicle screws without cement augmentation. Taking the advantage of osteointegration through the radial holes/slots was the main hypothesis for the study.

Table 3

Pull out test results. Comparison table of artificial fusion and cement augmentation

\begin{tabular}{|c|c|c|c|c|c|c|c|c|}
\hline \multirow[t]{3}{*}{ Sample ID } & \multicolumn{2}{|c|}{ Pull-out (Grade 40) } & \multicolumn{2}{|c|}{ Pull-out (Grade 40) } & \multicolumn{2}{|c|}{ Pull-out (Grade 10) } & \multicolumn{2}{|c|}{ Pull-out (Grade 10) } \\
\hline & Max. & St.D. & Max. & St.D. & Max. & St.D. & Max. & St.D. \\
\hline & \multicolumn{2}{|l|}{ Force $(\mathrm{N})$} & \multicolumn{2}{|l|}{ Force $(\mathrm{N})$} & \multicolumn{2}{|l|}{ Force $(\mathrm{N})$} & \multicolumn{2}{|l|}{ Force $(\mathrm{N})$} \\
\hline Solid Core & 671 & 60 & 671 & 60 & 236 & 17.4 & 236 & 17.4 \\
\hline $\mathrm{S} 2 \mathrm{H}$ & 1586 & 400 & 3034 & 232.9 & 978 & 258 & 432 & 46.9 \\
\hline $\mathrm{D} 2 \mathrm{H}$ & 2784 & 407 & 2795 & 20.5 & 787 & 163 & 498 & 22.9 \\
\hline $\mathrm{S} 3 \mathrm{H}$ & 1450 & 388 & 2782 & 262.4 & 919 & 243 & 511 & 52.8 \\
\hline D3H & 3381 & 146 & 2768 & 107.8 & 1095 & 151 & 492 & 19.6 \\
\hline SS & 2228 & 625 & 3104 & 98.08 & 954 & 146 & 491 & 23.6 \\
\hline \multirow[t]{2}{*}{ DS } & 3031 & 229 & 2936 & 170.3 & 1057 & 206 & 462 & 16 \\
\hline & \multicolumn{2}{|c|}{ Artificial Fusion } & \multicolumn{2}{|c|}{ Cement Augmented } & \multicolumn{2}{|c|}{ Artificial Fusion } & \multicolumn{2}{|c|}{ Cement Augmented } \\
\hline
\end{tabular}


Artificial fusion process was played a critical role while defining the post-fusion pullout performances of screws.

Chen et al. [22] studied pullout and torsion forces in a couple of designs. Chen mentioned the critical role played by radial holes for cement leakage into the spinal canal. The risk of cement leakage increases, when the holes are too proximal. Furthermore, the position of side holes is more essential for pullout strength than number of holes.

$\mathrm{S} 2 \mathrm{H}$ screws were pulled out at $1586 \mathrm{~N}$ after fusion, while a similar one, $\mathrm{S} 3 \mathrm{H}$ screws, and were pulled out at $1450 \mathrm{~N}$. In the meantime, SS screw pulled out at $2228 \mathrm{~N}$. Furthermore, measurements after osteoporotic (grade 10 synthetic PU foam) fusion revealed pullout results for D3H and DS fusion as $1095 \mathrm{~N}$ and $1057 \mathrm{~N}$, respectively. Pare et al. investigated pullout strength in cannulated screws, and reported the highest pullout strength as $572 \mathrm{~N} \pm 274 \mathrm{~N}$ [23]. This figure corresponded to half of the pullout strength obtained for grade $40 \mathrm{PU}$ foam used cases, while it was lower than the pullout strengths obtained for all grade 10 PU foam used cases of this study. Chen et al. [22] reported $320 \mathrm{~N}$ as an average value obtained for non-fusion pullout tests applied on foam, which is three times lower than the best performance obtained for screw design without cement augmentation in our study. As regards comparison to the expandable screws, Wu et al. [15] obtained maximum pullout strength of $1200 \mathrm{~N}$ in osteoporotic human cadaveric spine with cement augmented expandable screws. We obtained $1095 \mathrm{~N}$, which is higher than that obtained for expandable screws without cement augmentation, solid cored screws and PMMA cannulated screws in Wu's study. Becker et al. [13] conducted a similar study in osteoporotic human cadaveric spine. They compared PMMA balloon kyphoplasty to vertebroplasty. The screw pullout strength in solid core screwing in vertebroplasty measured $920 \mathrm{~N}$. This is $20 \%$ lower than the pullout strength obtained after artificial fusion in our study. Evans et al. [13] conducted a study on frozen bovine vertebrae. Their results reported an increase in pullout strength, from $1203 \mathrm{~N}$ to $1970 \mathrm{~N}$, after PMMA augmentation. Our new design showed increasing results for pullout strength of screws without cement augmentation, from $671 \mathrm{~N}$ to $3381 \mathrm{~N}$, in healthy bone after fusion. Some researchers studied calcium based cement in cannulated screws as an alternative to PMMA. In their study, Gao et al. [6] found that the pullout strength for cannulated screws with calcium apatite injection was $995 \mathrm{~N}$ in the osteoporotic spine. This figure is $10 \%$ lower than the best result achieved in our design without cement augmentation. Rohmiller et al. [24] compared pullout strengths on human cadaveric spine after calcium sulphate and PMMA injection, and reported that the highest pullout strength for PMMA was $1320 \mathrm{~N}$, whereas this was $1105 \mathrm{~N}$ for calcium sulphate injection. Our new design is as strong as calcium phosphate after artificial fusion process. This is very close to our results measured for the foam modeling healthy bone. Based on this, we can conclude that our design has obtained $3381 \mathrm{~N}$ with the foam modeling healthy bone (grade $40 \mathrm{PU}$ foam), which is approximately three times higher than the strength obtained by Rohmiller with calcium sulphate injected screws. Moreover, Hasemi et al. [25] conducted a multi-comparison in their synthetic foam. They studied the pullout strength of several cannulated screw designs on foam. Highest pullout strengths were recorded as $861 \mathrm{~N}$ with PMMA and $688 \mathrm{~N}$ with solid core screw without cement augmentation. This was found as $671 \mathrm{~N}$ in our study.

All these studies indicated that pullout strength increased in cannulated screws without cement augmentation, when fusion took place. Cement augmentation is a very common and effective practice. Screw pullout is more common during the early phase, when fusion has not yet taken place. However, our study indicated that cannulated screws without cement augmentation is an alternative to solid cored screws for healthy bone cases.

\section{Conclusion}

This study showed that using cannulated screws without cement augmentation for the cases with healthy bone can be a reliable alternative to solid cored screws. To the knowledge of investigators this is the first study comparing pullout strength for several cannulated screw designs after the artificial fusion process. The main limitation of this study was using living tissues for the more realistic results. However, using synthetic foams is accepted in literature to decrease the bone quality bias. In addition, finite element analysis can be carried out for such study. The number of tests and design parameters are highly enough for such a study. The finite element analysis and further investigations of designs can be a future work of this study. 


\section{Acknowledgments}

The author would like to acknowledge Turkish Science Foundation (TUBITAK) with the project \#111M583 and \#113S101 for the financial support for the study. Author also would like to acknowledge to the Osimplant (Bone implant) Ltd. Sti. for preparing the designed implants. Special thanks to Prof. Dr. Adem Şahin for his valuable comments on the study.

\section{References}

[1] J.D. Bartleson and H. Gordon Deen, Spine disorders medical and surgical management, Cambridge: Cambridge University Press, 2009.

[2] C.A. Lill, E. Schneider, J. Goldhahn, A. Haslemann and F. Zeifang, Mechanical performance of cylindrical and dual core pedicle screws in calf and human vertebrae, Arch Orthop Trauma Surg 126 (2006), 686-694.

[3] T. Demir, N. Camuşcu and K. Türeyen, Design and biomechanical testing of pedicle screw for osteoporotic incidents, Proc I Mech E P: H 226 (2012), 256-262.

[4] M.H. Krenn, W.P. Piotrowski, R. Penzkofer and P. Augat, Influence of thread design on pedicle screw fixation, $J \mathrm{Neu}$ rosurg Spine 9 (2008), 90-95.

[5] H. Mehtaa, E. Santosb, C. Ledoniob, J. Sembranob, A. Ellingsona and P. Pare, Biomechanical analysis of pedicle screw thread differential design in an osteoporotic cadaver model, Clinical Biomechanics 27 (2012), 234-240.

[6] M. Gao, W. Lei, Z. Wu, D. Liu and L. Shi, Biomechanical evaluation of fixation strength of conventional and expansive pedicle screws with or without calcium based cement augmentation, Clinical Biomechanics 26 (2011), 238-244.

[7] S. Vishnubhotla, W.B. McGarry, A.T. Mahar and D.E. Gelb, A titanium expandable pedicle screw improves initial pullout strength as compared with standard pedicle screws, The Spine Journal 11 (2011), 777-781.

[8] S. Wan, W. Lei, Z. Wu, D. Liu, M. Gao and S. Fu, Biomechanical and histological evaluation of an expandable pedicle screw in osteoporotic spine in sheep, Eur Spine $J 19$ (2010), 2122-2129.

[9] B. Bostan, I. Esenkaya, T. Gunes, M. Erdem, M. Asci and M.H. Kelestemur, A biomechanical comparison of polymethylmethacrylate-reinforced and expansive pedicle screws in pedicle-screw revisions, Acta Orthop Traumatol Turc 43 (2009), 272-276.

[10] W. Lei and Z. Wu, Biomechanical evaluation of an expansive pedicle screw in calf vertebrae, Eur Spine J 15 (2006), 321-326.

[11] S.D. Cook, J. Barbera, M. Rubi, S.L. Salkeld and T.S. Whitecloud 3rd, Lumbosacral fixation using expandable pedicle screws: An alternative in reoperation and osteoporosis, The Spine Journal 1 (2001), 109-114.

[12] O. Yaman, T. Demir, A.K. Arslan, M.A. İyidiker, T. Tolunay, N. CamuŞçu and M. Ulutaş, On the pullout strength comparison of various pedicle screw designs on synthetic foams and ovine vertebrae, Turk Neurosurg (ISI) (2013), DOI: 10.5137/1019-5149.JTN.8907-13.1.

[13] S.L. Evans, C.M. Hunt and S. Ahuja, Bone cement or bone substitute augmentation of pedicle screws improves pull-out strength in posterior spinal fixation, Materials in Medicine $\mathbf{1 3}$ (2002), 1143-1145.

[14] S. Becker, A. Chavanne, R. Spitaler, K. Kropik, N. Aigner and M. Ogon, Assessment of different screw augmentation techniques and screw designs in osteoporotic spines, Eur Spine J 17 (2008), 1462-1469.

[15] Z.X. Wu, M.X. Gao, H.X. Sang, Z.S. Ma, G. Cui and Y.Zhang, Surgical treatment of osteoporotic thoracolumbar compressive fractures with open vertebral cement augmentation of expandable pedicle screw fixation: A biomechanical study and a 2-year follow-up of 20 patients, Journal of Surgical Research 173 (2012), 91-98.

[16] M. Yazu, A. Kin, R. Kosaka, M. Kinoshita and M. Abe, Efficacy of novel-concept pedicle screw fixation augmented with calcium phosphate cement in the osteoporotic spine, J Orthop Sci 10 (2005), 56-61.

[17] S.D. Cook, S.L. Salkeld, T. Stanley, A. Faciane and S.D. Miller, Biomechanical study of pedicle screw fixation in severely osteoporotic bone, The Spine Journal 4 (2004), 402-408.

[18] A.K. Arslan, T. Demir, F. Örmeci, E. İnce and M.F. Toraman, Biomechanical performance of various cement augmented cannullated pedicle screw designs for osteoporotic bones, 15th International Conference on Advances in Materails Procecing Technologies (2012), pp. 22-26, Wollongong, Austuralia.

[19] ASTM F1839. Standard specification for rigid polyurethane foam for use as a standard material for testing orthopedic devices and instruments.

[20] K. Arslan, T. Demir, M.F. Ormeci, N. Camuscu and K. Tureyen, Post fusion pullout strength comparison of a novel pedicle screw with classical pedicle screws on synthetic foams, IMECH-E part H: Engineering in Medicine 227-2 (2013), 114-119.

[21] ASTM F543. Standard Specification and Test Methods for Metallic Medical Bone Screws.

[22] L.H. Chen, C.L. Tai, P.L. Lai, D.M. Lee, T.T. Tsai and T.S. Fu, Pullout strength for cannulated pedicle screws with bone cement augmentation in severely osteoporotic bone: Influences of radial hole and pilot hole tapping, Clinical Biomechanics 24 (2009), 613-618.

[23] P.E. Paré, J.L. Chappuis, R. Rampersaud, A.O. Agarwala, J.H. Perra and S. Erkan, Biomechanical evaluation of a novel fenestrated pedicle screw augmented with bone cement in osteoporotic spines, Spine (Phila Pa 1976) 36 (2011), E1210-4.

[24] M.T. Rohmiller, D. Schwalm, R.C. Glattes, T.G. Elalayli and D.M. Spengler, Evaluation of calcium sulfate paste for augmentation of lumbar pedicle screw pullout strength, The Spine Journal 2 (2002), 255-260.

[25] A. Hashemi, D. Bednar and S. Ziada, Pullout strength of pedicle screws augmented with particulate calcium phosphate: An experimental study, The Spine Journal 9 (2009), 404-410. 

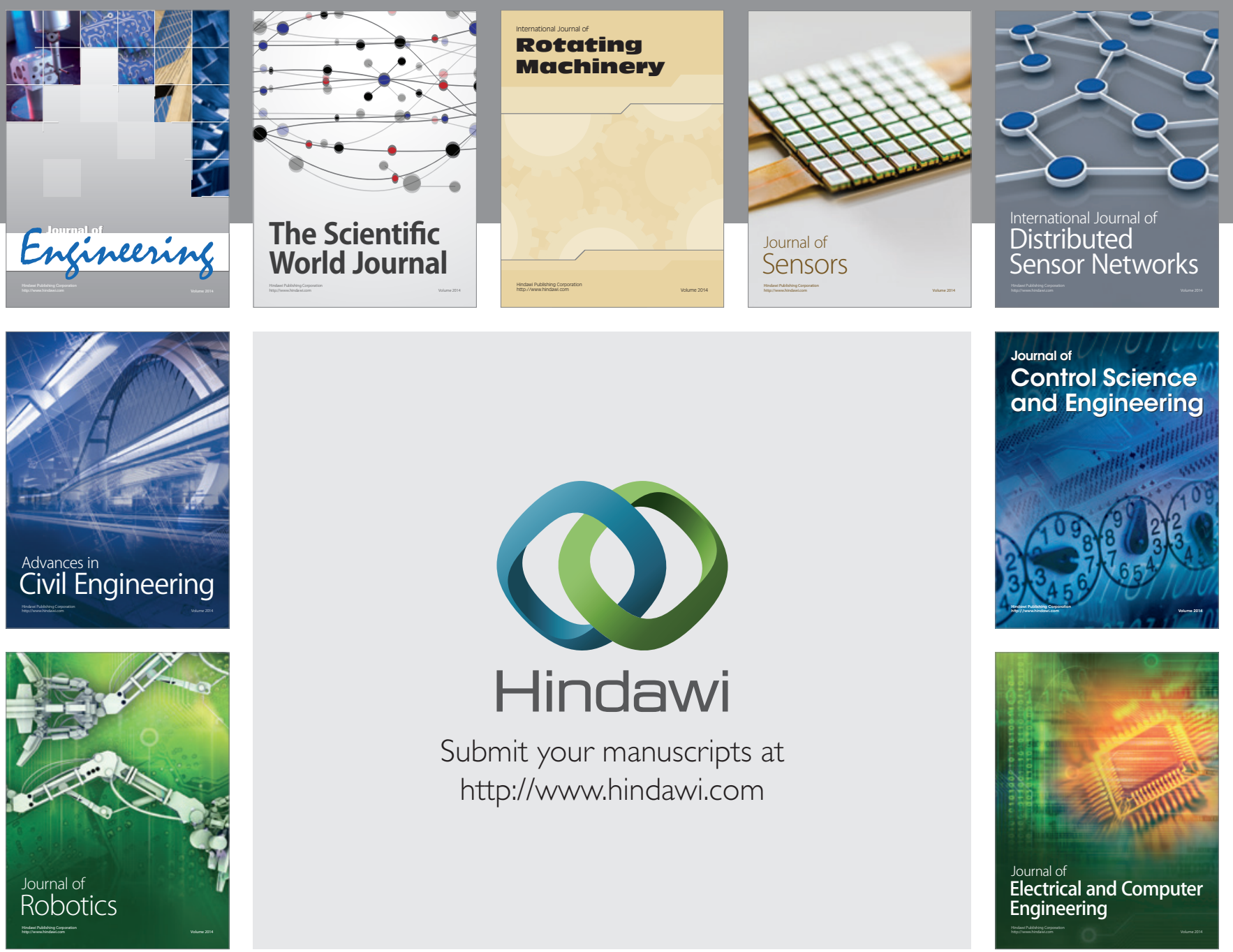

Submit your manuscripts at

http://www.hindawi.com
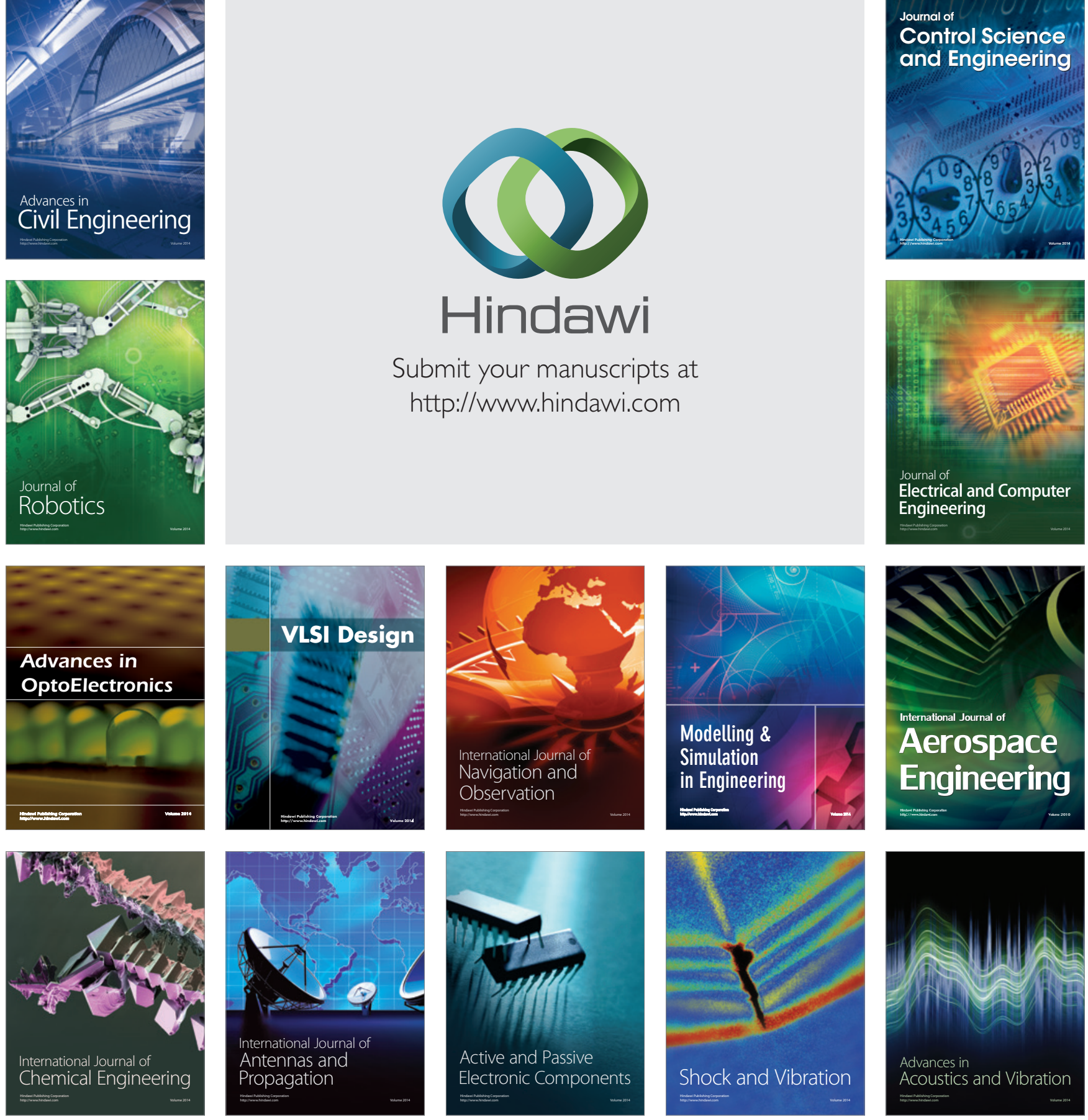